\title{
Open innovation at the Abbe School of Photonics
}

Christian Helgert, Reinhard Geiss, Stefan Nolte, Falk Eilenberger, David Zakoth, et al.

Christian Helgert, Reinhard Geiss, Stefan Nolte, Falk Eilenberger, David Zakoth, Oliver Mauroner, Thomas Pertsch, "Open innovation at the Abbe School of Photonics," Proc. SPIE 10452, 14th Conference on Education and Training in Optics and Photonics: ETOP 2017, 1045228 (16 August 2017); doi: $10.1117 / 12.2269953$

SPIE Event: 14th Conference on Education and Training in Optics and Photonics, ETOP 2017, 2017, Hangzhou, China 


\title{
Open Innovation at the Abbe School of Photonics
}

\section{Christian Helgert $^{\mathrm{a}^{\mathrm{*}}}$, Reinhard Geiss ${ }^{\mathrm{a}, \mathrm{b}}$, Stefan Nolte ${ }^{\mathrm{a}, \mathrm{b}}$, Falk Eilenberger ${ }^{\mathrm{c}}$, David Zakoth ${ }^{\mathrm{d}}$, Oliver Mauroner ${ }^{\mathrm{d}}$, and Thomas Pertsch ${ }^{\mathrm{a}, \mathrm{b}}$}

\author{
${ }^{a}$ Abbe Center of Photonics and Abbe School of Photonics, Friedrich Schiller University Jena, Albert-Einstein-Str. 6, 07745 Jena, \\ Germany \\ ${ }^{\mathrm{b}}$ Institute of Applied Physics, Friedrich Schiller University Jena, Max-Wien Platz 1, 07743 Jena, Germany \\ ${ }^{c}$ Fraunhofer Institute of Applied Optics and Precision Engineering, Albert-Einstein-Str. 9, 07745 Jena, Germany \\ ${ }^{\mathrm{d}}$ School of Business, University of Applied Sciences Mainz, Lucy-Hillenbrand-Str. 2, 55128 Mainz, Germany \\ * christian.helgert@uni-jena.de, Phone +49 3641 9-47960, www.asp.uni-jena.de and www.acp.uni- \\ jena.de
}

\begin{abstract}
The Abbe School of Photonics (ASP) provides and coordinates the optics and photonics education of graduate and doctoral students at the Friedrich Schiller University in Jena, Germany. The internationalized Master's degree program is the key activity in training students in the optical sciences. The program is designed to provide them with the skills necessary to fill challenging positions in industry and academia. Here, an essential factor is ASP's close collaboration with more than 20 German photonics companies. To sustain these partners' future economic development, the availability of highly qualified employees is constantly required. Accordingly, these industrial partners, the European Union, the local state and the federal German government are strongly involved in the sustainable development of ASP's curriculum by both conceptual and financial engagements. The main goal is to promote the students' academic careers and job experience in the photonics industry as well as in academia. To open up the program to students from all over the world, all ASP lectures and courses are taught in English. ASP's qualification strategy is fully research-oriented and based on the principles of academic freedom, competitive research conditions and internationalization at all levels. The education program is complemented by a structured doctoral student support and a prestigious guest professorship program. Recently, ASP and partners have started a project to build an open photonics laboratory in order to foster innovative and co-creative processes. The idea follows well-established open innovation schemes e.g. in electronics. This Photon Garage (German: "Lichtwerkstatt") will bring together professionals and interested laymen from different backgrounds to approach pertinent challenges in photonics. Here, we will share our latest insights into the potentials and opportunities offered by this novel educative approach.
\end{abstract}

Keywords: Photonics, optics, Master degree, doctoral studies, open innovation, industry support, internationalization

\section{INTRODUCTION - UNIFYING RESEARCH AND EDUCATION}

In Jena, the roots for an exceptional scientific community specializing in optics and photonics were laid by the breakthrough works of Ernst Abbe, Carl Zeiss and Otto Schott in the late $19^{\text {th }}$ century, rendering Jena the "cradle of modern optics". Due to this tradition, the Friedrich Schiller University Jena has a strong focus on photonics, which nowadays is concentrated in its interfaculty center Abbe Center of Photonics (ACP). This center hosts the research and education activities on optics and photonics of the university and incorporates major contributions from Jena's nonuniversity optical research institutes and photonics industry. Counting about 50 faculty members from physics, chemistry, biology and medicine, ACP shapes the future of the local photonics community by serving as lead partner in multiple collaborative research and education projects of national and international priority. ACP's research covers fundamental and applied topics and generates annually about 450 publications in peer-reviewed international journals. However, the main goal is to produce synergetic effects between ACP's research institutes, its industrial partners, and its multiple international collaborators in order to enable a scientific and economical added-value. This goal is explicitly focused both in research and education, which are inseparably cross-linked (see Figure 1).

14th Conference on Education and Training in Optics and Photonics: ETOP 2017, edited by Xu Liu,

Xi-Cheng Zhang, Proc. of SPIE Vol. 10452, 1045228 · @ 2017 ICO, IEEE, OSA, SPIE

CCC code: $0277-786 X / 17 / \$ 18 \cdot$ doi: $10.1117 / 12.2269953$ 


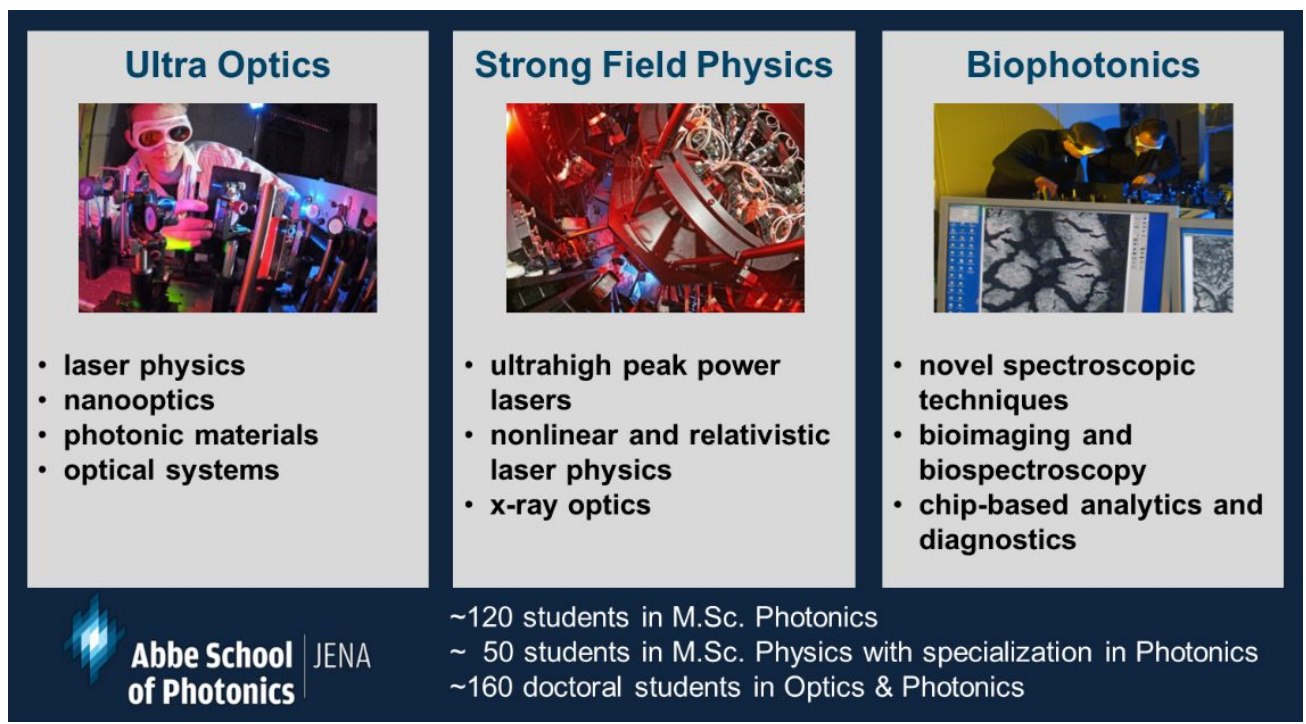

Figure 1. Areas of research at the Abbe Center of Photonics and cross-linking photonics education at the Abbe School of Photonics.

By its integrated Abbe School of Photonics (ASP), the center provides high-level training of young scientists during all qualification phases, promoting their careers in academia and photonics industry. ASP's concept and philosophy aim at establishing Jena as one of Europe's leading educational centers in optics and photonics. ASP was shaped from the traditionally established, broad spectrum of teaching and research activities in light sciences at the Friedrich Schiller University Jena. The School offers outstanding opportunities for high levels of qualification at the undergraduate and graduate levels in the subjects of optics and photonics. The underlying qualification strategy is fully research-oriented and based on the principles of academic freedom, competitive research conditions and internationalization at all levels of education and research. On the one hand, ASP's training promotes and links career phases of young scientists in academia. On the other hand, the School strongly recognizes its responsibility that the majority of its graduates will continue a career in research-intensive companies. All of our competitive career development measures are therefore designed to lay the foundation for successful careers in academia and industry.

The School was founded in 2008 following a competitive call by the local state and the German federal government. Since then, these governmental bodies, together with Germany's photonics industry and the European Union, provided competitive financial support necessary to support this process. The close collaboration with industrial partners is one key factor of ASP's education program. To sustain these partners' future economic development, all partners are in close contact with the ASP management to secure the availability of highly qualified personnel on the labor market at the right time. The ASP graduates are just the candidates which the German photonics industry is looking for.

\section{MASTER'S DEGREE PROGRAM}

As one of its core activities, with the Master of Science in Photonics (M.Sc. Photonics), ASP offers a two-year Master's degree program whose lectures and courses are taught completely in English. The M.Sc. Photonics is an internationally recognized graduate degree, providing multidisciplinary coverage in the field of optics and photonics, from upstream scientific aspects in engineering and knowledge regarding major areas of business. The recruitment for this program is based on a global strategy to select the best-matching students from around the world. In sync with this undergraduate education program, the School has established a coordinated doctoral program (comparable to a PhD program in the USA) which is merged with the local strategic research programs. Further academic measures and career development work are supported by an advanced, hands-on optics training and a comprehensive scholarship program for deserving Master's degree and doctoral students. Along with its academic course program, ASP organizes and carries out strong joint activities on business-oriented education which are run in collaboration with industrial and other academic partners.

Historically, the ASP Master's degree program is rooted in the European educational system and was funded by the Erasmus Mundus scheme of the European Union since 2009. Combining the international experience of a consortium formed of nine top-ranked European and US universities with Jena's profound expertise in optics education, the now 
well-established international Master's degree program provides the students with a solid knowledge in optical science and technology and is well-suited to incorporate students of various professional and socio-cultural backgrounds.

Nowadays, the M.Sc. Photonics program is fully integrated into the study courses of the Friedrich Schiller University Jena. The key enabling infrastructure of the ASP Master's degree program is provided by the scientific and teaching staff of the Faculty of Physics and Astronomy, the Faculty of Chemical and Earth Sciences, and other faculties. These lecturers offer long-standing expertise in optics and photonics education, and our Master's degree program takes full advantage of this fact. At the same time, the program is embedded into the rich research environment of the ACP. Students obtain hands-on experience in methodology courses taking place in research-grade photonics laboratories accessing various facilities of the university's institutes, the local Fraunhofer Institute for Applied Optics and Precision Engineering, the local Leibniz-Institute of Photonic Technology, the Helmholtz Institute Jena, as well as those of some prominent industrial partners. Master's degree students enrolled at ASP benefit from the strong connections within the German photonics industry and their involvement in our study track. In recent years, ASP's German partner companies were Agilent Technologies, Berliner Glas, Carl Zeiss AG, Edmund Optics, Jenoptik AG, Karl Storz, Leica, Linos QiOptiq, Lissotschenko Mikrooptik, Nanoplus, Novaled, Osram, Philips, Polytec, Precitec, Rofin, Saueressig, SICK AG, Trumpf, U2T Photonics and Wetzel Holding AG. Every graduate student experiences at least one mandatory internship with a focus on practical training in the application-oriented optics laboratories of these partner companies or at university institutes. Moreover, the final thesis of the Master's degree program can be fully accomplished through the successful completion of an industrial project.

Applying to the M.Sc. Photonics program is facilitated by a convenient online application system. All applicants are evaluated by ASP's selection committee with respect to their academic excellence, their English skills, their expressed intentions to pursue a career in Germany or Europe, and their previous qualifications; the top 60 to 100 students are selected to be eligible. In addition, comprehensive financial support is offered to the most excellent students through two-year scholarships funded by the German Federal Ministry of Education and Research (BMBF) and the local state government. Since October 2009, an annual average of 50 students out of about 700 annual applicants has been accepted at ASP. The student numbers with respect to their countries of origin is displayed in Figure 1. Since 2009, a total of more than 350 graduate students from about 50 different countries, with the highest numbers from central Asia and central Europe, have been enrolled at the School. Most notably, almost $90 \%$ of the graduate students are of non-German nationality. Likewise, the faculty of the School provides the main teaching activities of the Master of Science in Physics (M.Sc. Physics) program, whose students can choose to attend numerous special lectures in photonics. Currently, about 50 M.Sc. Physics with a specialization in photonics are enrolled. While approximately $80 \%$ of our graduate students, who successfully finish the program, continue on the academic track, accepting $\mathrm{PhD}$ positions at top-ranking universities worldwide, the other alumni regularly find appropriate positions in the photonics industry, strongly represented in Jena and also prevalent throughout Germany.

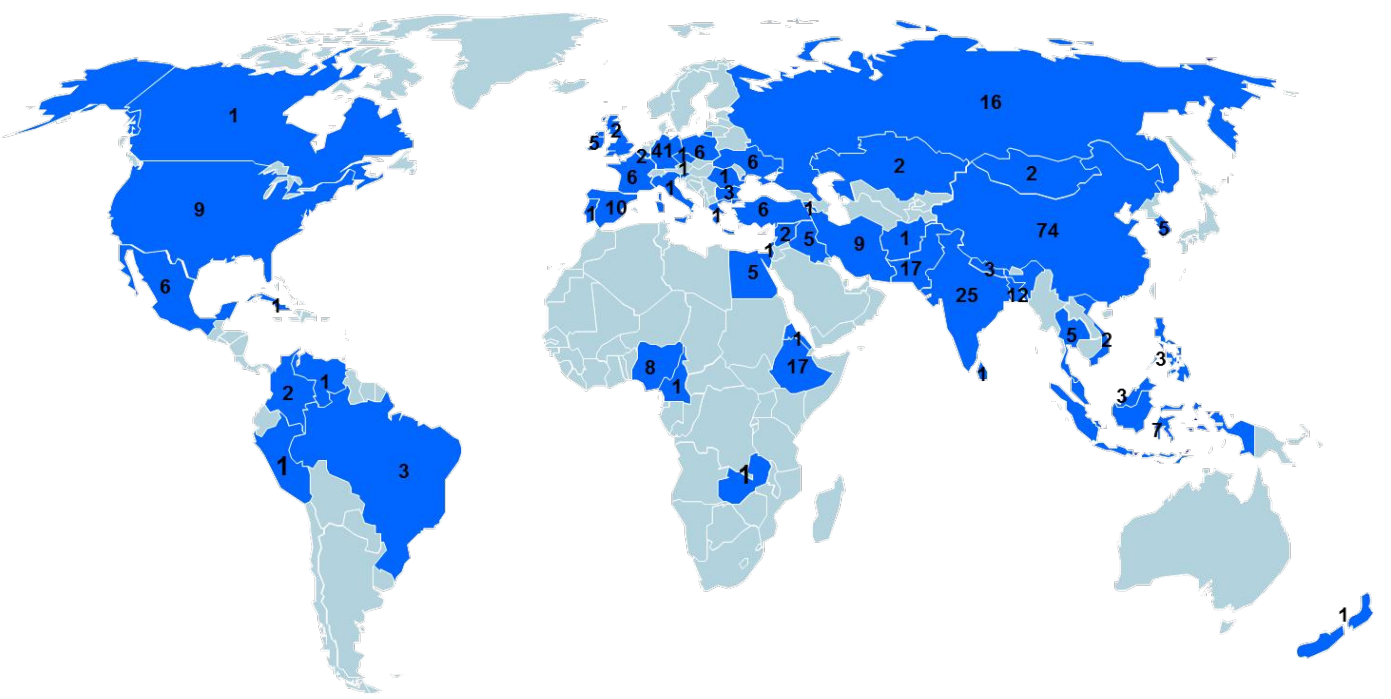

Figure 2. Countries of origin and M.Sc. Photonics student numbers since 2009. 


\section{DOCTORAL PROGRAM}

Incorporating the rich tradition of optics and photonics research, the doctoral program of the ASP (comparable with a $\mathrm{PhD}$ program in the USA) has now established sustainable structures for the education and interconnection of young academics in photonics. Since its start in 2009, ASP's doctoral program has been denoted by a constantly growing number of doctoral students pursuing research topics in optics and photonics at the Friedrich Schiller University, reflecting the successful acquisition of funding by its faculty. Currently, about 150 doctoral students have taken the opportunity to officially register in the ASP doctoral program. While a majority of the students who join the ASP doctoral program have a physics background, also chemists, biologists, and mathematicians perform their research projects at 11 different institutes and under the umbrella of the ASP doctoral program, following both the core optical disciplines and adjacent fields, such as e.g. nanochemistry or material sciences. Funding of the ASP doctoral program is provided by different sources, particularly by the supervising ACP principal scientists through various schemes of competitive third-party funding programs. The School functions as the umbrella organization for many of such projects, to strengthen the aspects of doctoral education along with individual research.

The selection and acquisition of new outstanding doctoral students is facilitated by choosing among the most excellent alumni of M.Sc. Photonics and M.Sc. Physics from Jena as well as hundreds of external applications, which we receive each year from students from the entire world. Currently, a share of $25 \%$ of ASP doctoral students is of foreign origin. Emphasizing its strong engagement in the further internationalization of studies, ASP is dedicated to this important primary aim of the institutional strategy of the whole university.

A cornerstone of the ASP philosophy is to regard and value our doctoral students as scientists in all respects. Most notable is the conscious and deep involvement of our doctoral students in top-notch research environments. Providing them with full access to the laboratories, they can more readily contribute their individual scientific contributions to globally prevailing research questions. During the work on their individual research project, a maximum of freedom to develop their own ideas and to follow personal scientific interests is allowed of every doctoral student. ASP's long-term experience has clearly shown that this philosophy translates into a timely advancement in self-reliance and individual responsibility of our students, which is essential for the development of their individual scientific careers at higher levels. To further promote the ASP doctoral students' scientific skills, participation in international conferences and presentation of their own contributions is encouraged and enabled, whenever possible. Already in the early stage of their doctoral phase, ASP students can realize their individual professional and personality-forming experiences on international platforms.

Both opportunities and particular challenges for the doctoral candidates are gained through an interdisciplinary, Englishspoken ASP doctoral seminar. Biweekly, two doctoral students present their research to a larger audience encompassing doctoral students, ASP supervisors, and interested guests. This scheme offers an ideal platform for the students to receive concentrated feedback from fellow students and professors, to possibly shift the student's perspective on their work disclosing formerly disregarded aspects, and to prepare for competitive, demanding on-stage-performances as young academics. The prototypical curriculum performed by an international student, who firstly enters in the M.Sc. Photonics and then successfully accomplishes his or her doctoral studies, is shown in Figure 3. 


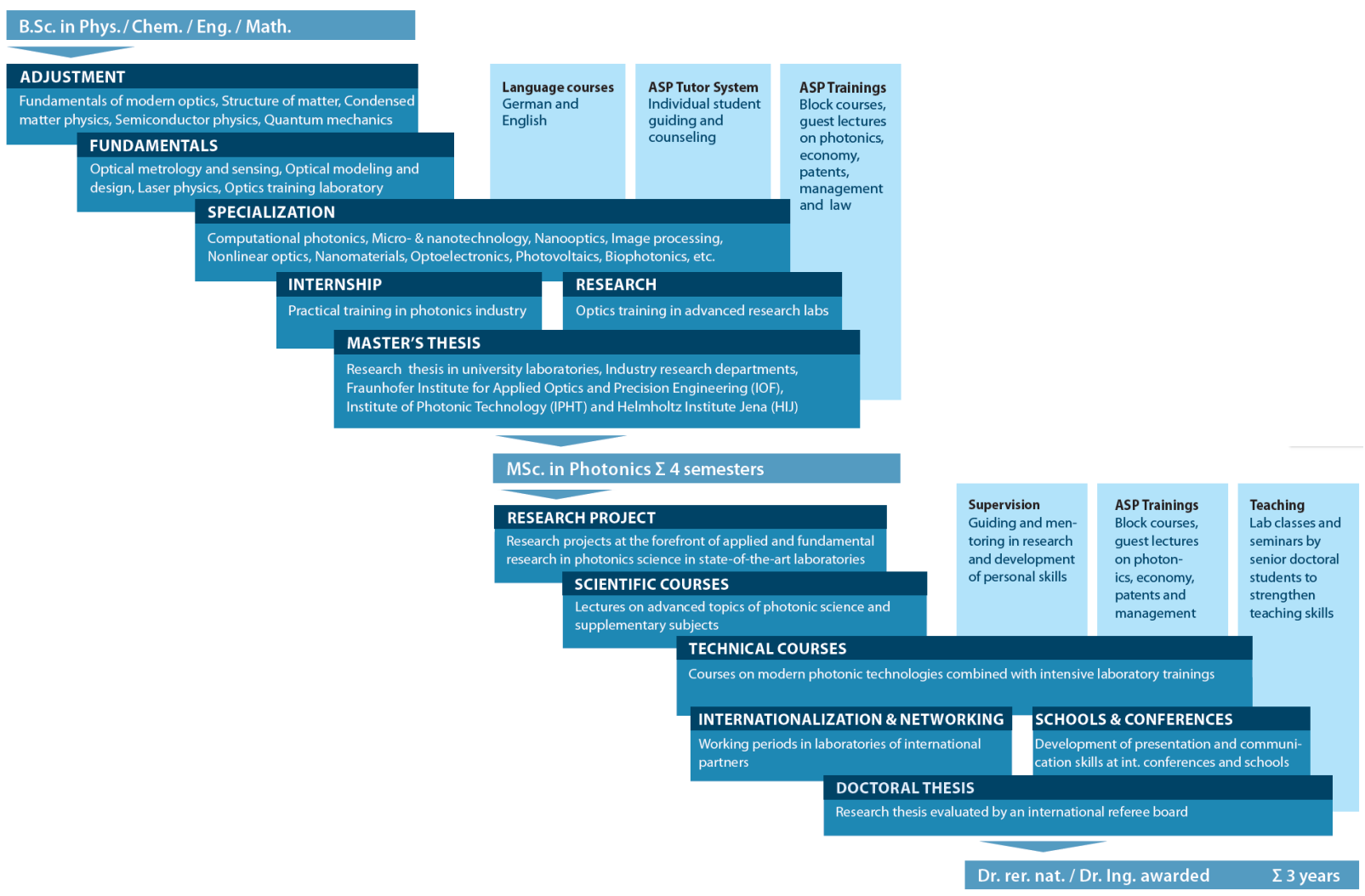

Figure 3. Curriculum of a prototypical two-year M.Sc. Photonics study track, followed by consecutive, three-year doctoral studies at the Abbe School of Photonics.

\section{OPEN PHOTONICS INNOVATION - THE PHOTON GARAGE}

Supported by an Open Photonics initiative of the German Federal Ministry of Education and Research (BMBF), we have recently set up a novel approach tackling open innovation processes which will be implemented in our regular photonics education. Open innovation processes are already established in different branches and sectors as a complementary asset to regular top-down business policies, but there is no comparable approach in photonics so far. In Jena and Mainz, this challenge will now be taken on by our so-called "Photon Garage" (in German: "Lichtwerkstatt"). This three-year-project is supposed to generate new forms of collaborations between companies, universities and students in order to involve a broad variety of people in innovation processes of photonics. For example, we are currently building up and host the first and so far only photonics makerspace in Germany. In addition to employees of the companies and scientists, especially students of our Master's degree programs, IT scientists and media managers are invited and encouraged to be the main innovative drivers of this process. The Photon Garage will include three key aspects:

- Open Photonics Makerspace: The Open Photonics Makerspace will appear as an open innovation laboratory, as a creative place where the exchange of ideas as well as shared projects are focussed. In the Makerspace, all stakeholders addressed will get access to various technologies and toolkits for generating, developing and testing ideas. By doing so, we address users ability to implement and execute their own ideas and to generate so-called user-driven innovation. The interdisciplinary and open approach of the Makerspace enables a new way of learning for the users and access to a broad innovation potential for enterprises. Bringing together users and professionals of the photonics industry will push the enterprises to develop their products more customercentered. This may evolve as a competitive advantage facing budget and time restrictions of small and medium sized companies (SMEs) within the photonics industry.

The Makerspace will be established in a spacious optical laboratory inside a modern research facility (see Figure 4). Similar to fablabs, the Makerspace will be accessible for the participants several times per week. 
- Open Photonics Brainshops: In order to provide the Photon Garage with ideas for projects, workshops and similar events will be conducted regarding specific topics with direct and indirect reference to photonics. The name Open Photonics Brainshops already illustrates that the mental and creative potential of all stakeholders addressed, especially the students with their professional skills, should be referred to, stimulated and reconnected. The workshops are intended to be interactive, based on Design Thinking and User Centered Design. Lecturers from various departments will discuss challenges and limits of technologies and application areas with the participants. Amongst other aspects, with the ideas identified in the Brainshops, students get the possibility to work on them within their Master's thesis in cooperation with companies. First approaches will include digital photonics, LED applications, medical photonics, photonic sensors, optics in automotive and photonic smart wear.

- Open Photonics Lectures: These special lecture events take place in the Makerspace and complement the Brainshops, provide the opportunity to mediate methods of areas like innovation, innovations management, entrepreneurship and design to the different groups of players. The aim of these events is the improvement of the actors' basic skills with respect to generating ideas and innovation. By using events, well adapted for particular target groups (e.g. interactive workshops, contests), practice and skills are focussed. The selection of topics is therefore completely different to the topics of traditional lectures and seminars at university which leads to the enhancement of the experimental and entrepreneurial spirit/characteristic of the Open Photonics Lectures.
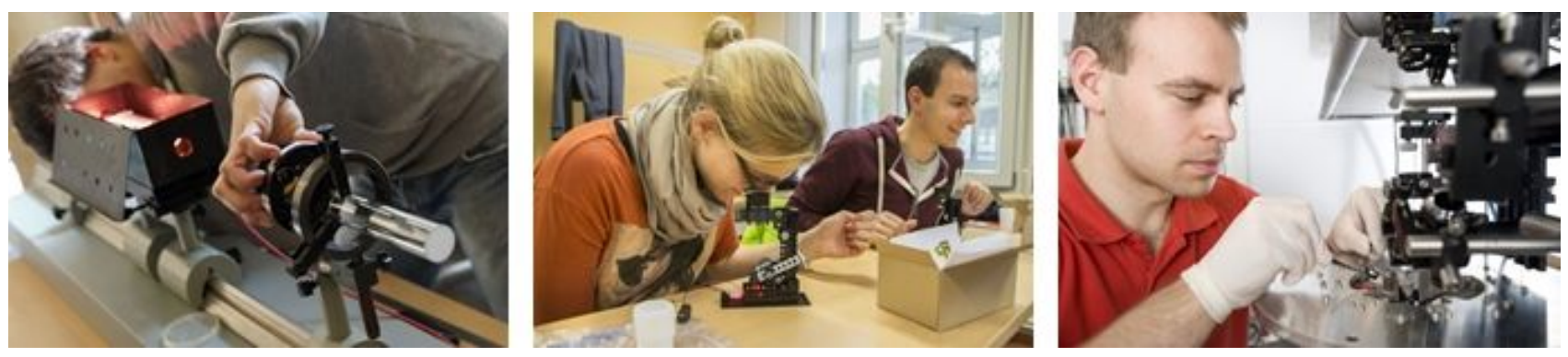

Figure 4. First hands-on experiments of professionals and laymen concerning open photonics innovation inside the new Photonics Makerspace.

\section{ACKNOWLEDGEMENTS}

$\mathrm{ACP}$ and ASP constantly acquire multiple sources of funding to establish and maintain its interconnected research and education program. The larger share of financial aid is for indirect support, attracted by ACP's principal scientists through many different competitive third-party funding schemes. Particularly, and on behalf of all colleagues, the authors wish to acknowledge financial support by the German Research Society (DFG) within the Collaborative Research Centers (CRC) TR7, TR18 and the International Research Training Group (IRTG) 2101, the European Union within "Photonics4life" (European network of excellence for biophotonics), the German Federal Ministry of Education and Research (BMBF) within the funding schemes "MaPho - Master education in Photonics", "Lichtwerkstatt - Open photonics innovation", "ZIK ultra optics" (Center for Innovation Competence for nanooptics and high-intensity laser physics), "Infectognostics" (public-private research campus on optical technologies in medicine) and "3Dsensation" (collaborative research initiative on human-machine-interaction in public-private partnership), the Thuringian state government within its two "ProExcellence" initiatives, the Carl Zeiss Foundation within its initiative on structural measures "Abbe School of Photonics", and the German Academic Exchange Service (DAAD) within its graduate scholarship programs. Moreover, ASP gratefully receives financial support from more than 20 German photonics companies, as detailed above. 